\title{
Deprescribing one year on: challenging the first iatrogenic epidemic
}

\author{
Nina Barnett, ${ }^{1}$ Doron Garfinke ${ }^{2,3}$
}

In January 2017 EJHP published a themed issue on the subject of deprescribing, a 'hot topic' for clinicians who are challenged with managing inappropriate medication use and polypharmacy. However, it was clear that, despite the enthusiasm of clinicians to manage this issue, there was a paucity of validated tools for use in practice and knowledge of the patient's view of deprescribing.

A literature search on this subject has revealed some interesting developments during 2016/7. Deprescribing in specific therapeutic areas and new deprescribing tools have been explored, as well as publications on both patient and general practitioner beliefs about and attitudes to deprescribing. In diabetes, medication for care home residents in the UK was reviewed using a medicines optimisation tool. The majority had been prescribed potentially inappropriate medication. Deprescribing was endorsed by the physician in over one-third of cases $39 \%$ of 106 residents). ${ }^{1}$

Another study involving deprescribing of benzodiazepines and ' $Z$ drugs' in primary care described how the switching rate was higher than the deprescribing rate when a tool was used to support review. ${ }^{2}$ Effective deprescribing in a haemodialysis unit was undertaken using a bespoke tool resulting in $88 \%$ of eligible patients $(n=41)$ having at least one medication deprescribed. ${ }^{3}$

The Tool to Reduce Inappropriate Medicines (known as TRIM), has been used to link electronic health records to clinical decision systems to improve communication about medication and prescribing in a clinic setting. While the tool supported better medicines reconciliation, it did not affect the number of

\footnotetext{
${ }^{1}$ Medicines Use and Safety Team, NHS Specialist Pharmacy Service, London North West Healthcare NHS Trust, Harrow, UK

${ }^{2}$ Geriatric Palliative Service, Edith Wolfson Medical Center, Holon, Israel

${ }^{3}$ Homecare Hospice Service, Israel Cancer Association, Givatayim, Israel
}

Correspondence to Professor Nina Barnett, Medicines Use and Safety, NHS Specialist Pharmacy Service, London North West Healthcare NHS Trust, Harrow HA1 3UJ, UK; nina.barnett@nhs.net medications or potentially inappropriate medications prescribed. ${ }^{4}$

There has been some development in understanding the patient view of deprescribing. Reeve and Hilmer from Australia have authored a number of papers using the Patient Attitudes Towards Deprescribing (PATD) questionnaire, which has been tested for validity and reliability. Their questionnaire has been used for older adults and caregivers in an Australian outpatient setting and an Italian inpatient setting. The results suggested that many older people would like to reduce the number of medicines they take and were less aware of reasons for medication. ${ }^{5}$ Key factors identified included perceived burden of medication taking, belief in appropriateness of medication use (harms and benefits), concerns about stopping the medication and level of involvement/knowledge of medications. ${ }^{6}$ Another study by Linsky et al identified four factors which include medication knowledge, concerns and importance as well as interest in stopping medication. ${ }^{7}$ New Zealand general practitioners were included in a study ${ }^{8}$ which explored their insight into deprescribing for the multimorbid older adult using semi-structured interviews and a hypothetical patient case. This work revealed a lack of clarity around deprescribing. The NICE multimorbidity guidelines go some way to address this for prescribers in the UK and support re-evaluation of the appropriateness of using single condition based prescribing guidelines in relation to the overall benefit to patients. The authors suggest that it is the role of the family doctor to discuss desprescribing with the patient as a routine part of clinical care, not just at the end of life

All the tools until now have focused on 'serial deprescribing'-that is, stopping medicines one at a time. This approach, however, is empirical and based on putative safety concerns rather than evidence. It is our belief that, in some instances, the risk of harm of continuing a number of medications can outweigh the benefit of using 'serial deprescribing' to decrease inappropriate medication use (IMU). In older people and/or in those in whom life expectancy is relatively short, one of the authors (DG) suggests that stopping one medicine at a time is neither practical nor even ethical. Given that the number of medications is the strongest predictor of IMU and that the number of drug interactions rises in proportion with increasing polypharmacy, the best cure for IMU may be deprescribing of as many medications as possible at the same time. Indeed, it is conceivable that, for patients taking a large number of medicines, the exact interactions causing the iatrogenic damage or symptoms are invisible and undetected.

Initial studies in a nursing home ${ }^{10}$ and in the community ${ }^{11}$ exploring 'poly-deprescribing' (PDP) that is, stopping more than one medicine at a time, were demonstrated to be both efficacious and safe. The recent longitudinal study by one of the authors (DG) compared PDP recommendations of $\geq 3$ medications to 177 older people. $^{12}$ Of the 122 who underwent PDP, the number of drugs was eventually reduced from an average of 10 medications at baseline to $\sim 3.8$. Fifty-five non-responders remained on 10 drugs or more $(\mathrm{P}=0.0001)$. At follow-up $\geq 3$ years later, functional, mental and cognitive status, appetite, sleep quality and 'major complications' were all significantly better in the intervention group; mortality and hospitalisations were comparable. Thus PDP was demonstrated to be safe and also to be associated with improved clinical outcomes and quality of life compared with the outcomes for comparable older people who take all medications based on all specialists' clinical guidelines.

It is important to be mindful of concerns around deprescribing, and a narrative review of safety concerns with deprescribing ${ }^{13}$ identified four categories of concern. These included withdrawal (adverse) effects, recurrence of condition, consequences of change to drug interactions and negative impact on the patient-doctor relationship. Mitigation requires a person-centred approach to deprescribing using a structured process which includes planned medication reduction and withdrawal with appropriate follow-up and monitoring. Recent publications, including that of one of the authors (DG), have contributed to the movement seeking to overcome the main professional barriers to deprescribingnamely, uncertainty regarding the effectiveness of deprescribing strategies, paucity of evidence-based guidelines and clinician fear of deprescribing-related harm.

While we have reached a point of awareness of the 'epidemic' of polypharmacy and its consequences, this is only the first crucial step. It is now time to take the second step where we mobilise forces internationally to manage the challenge. The International 
Group for Reducing Inappropriate Medication Use and Polypharmacy (IGRIMUP) has set the foundation for sharing effective interventions through creation of an alliance of practitioners and researchers. The challenge of deprescribing in preventative, curative and even palliative situations must all be addressed through education by including it as an integral part of the syllabus of doctors, pharmacists, nurses, health policy makers as well as in postgraduate continuing professional development. ${ }^{14}{ }^{15}$ Education should include methods of engaging with the patient, family and care-giver views, beliefs, attitudes and preferences, which are key to success. Public awareness of appropriate and inappropriate polypharmacy, including linking with the general press and social networks, will support a collaborative approach between clinicians and patients.

\section{Competing interests None declared.}

Provenance and peer review Not commissioned; internally peer reviewed.

(C) European Association of Hospital Pharmacists (unless otherwise stated in the text of the article) 2018. All rights reserved. No commercial use is permitted unless otherwise expressly granted.

$$
\text { Check for updates }
$$

To cite Barnett N, Garfinkel D. Eur J Hosp Pharm 2018:25:63-64.

Published Online First 27 January 2018

Eur J Hosp Pharm 2018:25:63-64.

doi:10.1136/ejhpharm-2017-001482

\section{REFERENCES}

1 Andreassen LM, Kjome RL, Sølvik UØ, et al. The potential for deprescribing in care home residents with type 2 diabetes. Int J Clin Pharm 2016:38:977-84

2 Roig SO, Gomez-Valent M, Trias LB, et al. Benzodiazepines and z-drugs deprescription by a multidisciplinary pharmacotherapy revision focused on the patient. Eur J Hosp Pharm 2017;24:A251.

3 McIntyre C, McQuillan R, Bell C, et al. Targeted deprescribing in an outpatient hemodialysis unit: a quality improvement study to decrease polypharmacy. Am J Kidney Dis 2017;70:611-8.

4 Fried TR, Niehoff KM, Street RL, et al. Effect of the Tool to Reduce Inappropriate Medications on medication communication and deprescribing. J Am Geriatr SoC 2017:65:2265-71.

5 Galazzi A, Lusignani M, Chiarelli MT, et al. Attitudes towards polypharmacy and medication withdrawal among older inpatients in Italy. Int J Clin Pharm 2016;38:454-61.

6 Reeve E, Low LF, Shakib S, et al. Development and validation of the Revised Patients' Attitudes Towards
Deprescribing (RPATD) questionnaire: versions for older adults and caregivers. Drugs Aging 2016;33:913-28.

7 Linsky A, Simon SR, Stolzmann K, et al. Patient perceptions of deprescribing: survey development and psychometric assessment. Med Care 2017;55:306-13.

8 Ailabouni NJ, Nishtala PS, Mangin D, et al. General practitioners' insight into deprescribing for the multimorbid older individual: a qualitative study. Int J Clin Pract 2016;70:261-76.

9 Guthrie B, Makubate B, Hernandez-Santiago V, et al. The rising tide of polypharmacy and drug-drug interactions: population database analysis 1995-2010. BMC Med 2015:13:74

10 Garfinkel D, Zur-Gil S, Ben-Israel J. The war against polypharmacy: a new cost-effective geriatric-palliative approach for improving drug therapy in disabled elderly people. Isr Med Assoc J 2007;9:430-4.

11 Garfinkel D, Mangin D. Feasibility study of a systematic approach for discontinuation of multiple medications in older adults: addressing polypharmacy. Arch Intern Med 2010;170:1648-54.

12 Garfinkel D. Poly-de-prescribing to treat polypharmacy: efficacy and safety. Ther Adv Drug Saf 2018;9:25-43.

13 Reeve E, Moriarty F, Nahas $\mathrm{R}$, et al. A narrative review of the safety concerns of deprescribing in older adults and strategies to mitigate potential harms. Expert Opin Drug Saf 2018;17:39-49.

14 Jubraj B, Marvin V, Poots AJ, et al. A pilot survey of junior doctors' attitudes and awareness around medication review: time to change our educational approach? Eur J Hosp Pharm Sci Pract 2015;22:243-8.

15 Poots AJ, Jubraj B, Barnett NL. Education around deprescribing: 'spread and embed' the story so far. European Journal of Hospital Pharmacy 2017;24:7-9. 